\title{
Pet-saúde interprofissionalidade: construindo ferramentas de educação em saúde no contexto da pandemia
}

Pet-health interprofessionality: building health education tools in the context of the pandemic

Interprofesionalidad pet-salud: construyendo herramientas de educación en salud en el contexto de

la pandemia

Brenda Beatriz Silva Monteiro ORCID: https://orcid.org/0000-0002-2682-3051 Universidade do Estado do Pará, Brasil

E-mail: bbeatrizfisio@gmail.com

Fabíola da Silva Costa

ORCID: https://orcid.org/0000-0002-2149-8499 Universidade do Estado do Pará, Brasil

E-mail: fabiolabraaz63@gmail.com

Dayanne de Nazaré dos Santos ORCID: https://orcid.org/0000-0002-6389-7287 Centro Universitário Maurício de Nassau, Brasil

E-mail: enfdayannesantos@yahoo.com.br

Marwim Luis Batista Costa

ORCID: https://orcid.org/0000-0002-3787-1761 Universidade do Estado do Pará, Brasi E-mail: marwimlbcosta@ hotmail.com

Erica Silva de Souza Matsumura ORCID: https://orcid.org/0000-0003-4844-8050 Universidade do Estado do Pará, Brasil E-mail: erica.s.souza@terra.com.br

Luciano Gil Saldanha Torres

ORCID: https://orcid.org/0000-0002-7327-7858

Universidade do Estado do Pará, Brasil

E-mail: lucianotorres10@gmail.com

Nayara Gabrielle de Lima Lobo ORCID: https://orcid.org/0000-0002-5735-2029 Universidade da Amazônia, Brasil E-mail: naylobo43@gmail.com

Maria Vitória Santos Briglia

ORCID: https://orcid.org/0000-0003-3765-0042 Universidade do Estado do Pará, Brasil E-mail: mavibriglia@gmail.com

Dayane Cereja Ferreira da Silva ORCID: https://orcid.org/0000-0002-7801-4811

Universidade do Estado do Pará, Brasil E-mail: dayanecereja01@ gmail.com

Milena da Conceição Aviz da Silva ORCID: https://orcid.org/0000-0002-5687-7245 Universidade da Amazônia, Brasil E-mail: milenaaviz15@gmail.com

Emilly Cássia Soares Furtado ORCID: https://orcid.org/0000-0002-9796-9859

Universidade Federal do Pará, Brasil

E-mail: emillycsoares15@gmail.com

Letícia Neris Macedo

ORCID: https://orcid.org/0000-0002-9974-3036

Centro Universitário Maurício de Nassau, Brasil E-mail: macedonleticia@hotmail.com

Maria Luisa Leite da Costa ORCID: https://orcid.org/0000-0003-4066-1180

Universidade da Amazônia, Brasil E-mail: malucoosstta@gmail.com

Sheila Souza de Freitas

ORCID: https://orcid.org/0000-0002-4239-5587

Universidade Federal do Pará, Brasil

E-mail: sheilafisio1212@gmail.com 
Raissa Helena Rodrigues Machado

ORCID: https://orcid.org/0000-0003-3129-6556 Universidade Federal do Pará, Brasil E-mail: raissahelen2@gmail.com

\begin{abstract}
Resumo
Objetivo: descrever as experiências vivenciadas por acadêmicos de saúde de uma Universidade Pública a cerca da construção de ferramentas de educação em saúde sobre covid-19. Metodologia: Relato de experiência, vivenciado a partir do projeto de extensão desenvolvido por uma Universidade Pública e a Secretaria Municipal de Saúde (SESMA) dentro do Programa de Educação pelo Trabalho para Saúde (PET-Saúde) Interprofissionalidade. Esta experiência foi desenvolvida durante a pandemia pela COVID-19, de forma virtual, entre os meses de março a outubro de 2020. Resultados: Visando o esclarecimento da população acerca da nova doença responsável pela pandemia em curso, a COVID-19, os alunos integrantes do projeto PET-Saúde 2019-2020, sob supervisão de seus preceptores e coordenadores, realizaram pesquisas e trocaram informações acerca da temática, trabalhando estas informações de forma interprofissional com a finalidade de fornecer à população geral um apanhado de informações que, ao mesmo tempo completas ao abrangerem as responsabilidades e competências dos cursos dos acadêmicos envolvidos. Conclusão: A cartilha favoreceu a disseminação de informações seguras, em um período turbulento e permeado de notícias falsas, buscou-se fontes confiáveis e embasamento científico prioritariamente durante todo processo, além do mais efetivando as práticas colaborativas, como eixo primordial do programa.
\end{abstract}

Palavras-chave: Educação interprofissional; Atenção básica; Comunidade; Pandemia.

\begin{abstract}
Objective: describe the experiences lived by health academics from a Public University about the construction of health education tools about covid-19. Methodology: Experience report, lived from the extension project developed by a Public University and the Municipal Health Secretariat (SESMA) within the Interprofessional Education through Work Program for Health (PET-Saúde). This experience was developed during the pandemic by COVID-19, virtually, from March to October 2020. Results: Aiming at clarifying the population about the new disease responsible for the current pandemic, COVID-19, students members of the PET-Saúde 2019-2020 project, under the supervision of their preceptors and coordinators, carried out research and exchanged information about the theme, working with this information in an interprofessional way in order to provide the general population with a collection of information that, at the same time complete by covering the responsibilities and skills of the courses of the academics involved. Conclusion: The booklet favored the dissemination of secure information, in a turbulent period and permeated with false news, reliable sources and scientific basis were sought primarily throughout the process, in addition to effecting collaborative practices, as the program's primary axis.
\end{abstract}

Keywords: Interprofessional education; Basic attention; Community; Pandemic.

\begin{abstract}
Resumen
Objetivo: describir las experiencias vividas por académicos de la salud de una Universidad Pública sobre la construcción de herramientas de educación en salud sobre covid-19. Metodología: Informe de experiencia, vivido desde el proyecto de extensión desarrollado por una Universidad Pública y la Secretaría Municipal de Salud (SESMA) dentro del Programa de Educación Interprofesional por el Trabajo para la Salud (PET-Saúde). Esta experiencia fue desarrollada durante la pandemia por COVID-19, virtualmente, de marzo a octubre de 2020. Resultados: Con el objetivo de esclarecer a la población sobre la nueva enfermedad responsable de la actual pandemia, COVID-19, estudiantes miembros del PET-Saúde 2019- El proyecto 2020, bajo la supervisión de sus preceptores y coordinadores, realizó investigaciones e intercambió información sobre el tema, trabajando con esta información de manera interprofesional con el fin de brindar a la población en general una recopilación de información que, al mismo tiempo, complete por cubriendo las responsabilidades y habilidades de los cursos de los académicos involucrados. Conclusión: El cuadernillo favoreció la difusión de información segura, en un período convulso y permeado de noticias falsas, se buscaron fuentes confiables y bases científicas principalmente durante todo el proceso, además de hacer efectivas las prácticas colaborativas, como eje principal del programa.
\end{abstract}

Palabras clave: Educación interprofesional; Atención básica; Comunidad; Pandemia.

\title{
1. Introdução
}

A Constituição Federal de 1988 e a Lei nº8.080 de 19 de setembro de 1990 (Lei Orgânica da Saúde) apresentam a noção de saúde como um direito de todos os cidadãos e dever que o estado deve assegurar. Esta última propõe como uma das competências do Sistema Único de Saúde (SUS) a garantia de formação para os profissionais da saúde (Brasil, 1988/2016; Agreli, Peduzzi \& Silva, 2016). 
Em 2003, foi instituída a Secretaria de Gestão no Trabalho e Educação na Saúde, que reafirmou a relevância da área de recursos humanos para a concretização da Política Nacional de Saúde. Esta secretaria foi criada para combater inúmeros desafios, incluindo os encontrados nos processos de formação, planejamento, gestão e regulação do trabalho em saúde para a efetivação do funcionamento do SUS (Brasil, 2011; Araújo, 2014).

Referente às políticas de reorientação da formação dos profissionais da saúde, as principais linhas voltam-se a reformulações da mudança no ensino nas graduações e para o desenvolvimento profissional dos trabalhadores de nível superior e técnico do SUS. Para compor estes segmentos, diversos programas e ações foram criados ao longo dos anos, desencadeando mudanças e fortalecimento na Política Nacional de Saúde (Batista, 2013; Cerballos, 2015).

Dessa forma, busca-se, com a Política de Educação em Saúde, promover ações que possam articular as competências individuais as demandas institucionais, que possam gerar potencial sustentável. Assim, o Ministério da Saúde tem destinado apoio técnico e financeiro para os programas, projetos e políticas públicas, cujos objetivos direcionam-se à qualificação e adequação do perfil dos profissionais às necessidades sociais dos serviços, tendo como eixo a integração ensino-serviço (Brasil; Farias, et al., 2018).

Buscando seguir esta linha, o Plano Nacional de Saúde (2020-2023) apresenta eixos e diretrizes divididos em 13 domínios, a serem seguidos pelo Poder Público juntamente com um processo de debates, instituindo assim o Conselho Nacional de Saúde (CNS) (Grillo, Ibarra \& Mezadri, 2019; Brasil, 2020).

Para tanto, foram estabelecidas estratégias que possam inserir os jovens no âmbito da formação para a saúde, dentre elas, temos o Programa de Educação pelo Trabalho para a Saúde (PET-Saúde), que propõe que os mesmos, estudem, pratiquem e pesquisem ações de qualificação de educação em saúde, nos serviços de saúde e atuem como promotores de mudanças nas graduações em saúde, direcionados pelas Diretrizes Curriculares e as necessidades do SUS e da população brasileira (Brasil, 2018).

O PET-Saúde, que apresenta como direcionamento a educação pelo trabalho, foi instituído por meio de uma parceria entre o Ministério da Saúde (MS) e o Ministério da Educação (MEC) no ano de 2008, com o objetivo inicial de fomentar grupos de aprendizagem tutorial no âmbito da Estratégia de Saúde da Família (ESF), sendo posteriormente estendido para outras áreas do SUS para atuar no combate de doenças, constituindo, nos dias atuais, uma das maiores estratégias para propor mudanças nos processos de educação e formação de estudantes e profissionais no SUS (Lourenço, et al., 2017).

Desta feita, o PET-Saúde (projeto 2019-2020) configura-se em uma das estratégias mais fortalecidas para a implementação da Educação Interprofissional (EIP), uma vez que vincula as atividades das universidades com as realizadas nos serviços de saúde e, desta vez, foram utilizados os pressupostos da EIP.

Nesse sentido, segundo a Organização Mundial da Saúde (2010), a Educação Interprofissional pode ser caracterizada como o aprendizado mútuo de duas ou mais profissões, tendo como finalidade promover/alcançar resultados positivos. Já as práticas colaborativas são a aplicação destes aprendizados direcionados às mais diversas ações em benefício do usuário.

No cenário nacional, o trabalho interprofissional com pressupostos das práticas colaborativas ainda é pouco difundido e aplicado. Por esse motivo, este estudo tem como objetivo descrever as experiências vivenciadas por acadêmicos de saúde de uma Universidade Pública a cerca da construção de ferramentas de educação em saúde sobre covid-19.

\section{Metodologia}

Trata-se de um estudo do tipo relato de experiência de caráter descritivo com abordagem qualitativa, vivenciado a partir do projeto de extensão desenvolvido por uma Universidade Pública e a Secretaria Municipal de Saúde (SESMA) dentro do Programa de Educação pelo Trabalho para Saúde (PET-Saúde) Interprofissionalidade do Edital No 10, 23/07/2018. Sendo 
assim, coordenadores, tutores, preceptores e alunos de diversas áreas de atuação pertencentes às referidas instituições têm múltiplas vivências e experiências em conjunto.

É oportuno destacar que o relato de experiência é um texto que descreve uma dada experiência que possa contribuir de forma relevante para sua área de atuação. É a descrição que um autor ou uma equipe fazem de uma vivência profissional tida como exitosa ou não, mas que contribua com a discussão para a melhoria do cuidado na saúde (Grillo, Ibarra \& Mezadri, 2019).

Assim, esta experiência foi desenvolvida durante a pandemia pela COVID-19 (Coronavirus Disease 2019, em tradução livre, “Doença do Coronavírus de 2019”), de forma virtual, entre os meses de março a outubro de 2020. Durante este período, foram realizados encontros semanais ou mensais com o grupo do PET- Saúde por meio de redes sociais como Whatsapp, Instagram, Plataforma Google Meet e YouTube.

O projeto PET-Saúde Interprofissionalidade (2019-2020) desta Universidade é formado por 5 grupos acadêmicos e profissionais de diversas profissões, com atuação no Distrito da Sacramenta em Belém/PA.

Ademais, os fundamentos teórico-conceituais e metodológicos do Edital nº10 - 2019/2020 propõe:

1. Educação Interprofissional;

2. Trabalho Colaborativo;

3. Trabalho em equipe entendido como processo permanente de colaboração;

4. Autocuidado e a autonomia das pessoas, famílias, grupos e comunidades.

O desenvolvimento deste relato ocorreu em etapas, sendo estas:

\section{$1^{o}$ Etapa - Embasamento teórico:}

Foi desenvolvido com base na investigação de publicações científicas sobre a temática, possuindo como suporte teórico os arquivos científicos resgatados nas bases de dados: Scientific Eletronic Library Online (SciELO), Portal de Periódicos CAPES/MEC, base LiLACS (Literatura Latino Americana e do Caribe em Ciências da Saúde) e PUBMED (National Library of Medicine - NLM).

O levantamento bibliográfico inicial foi executado durante os meses de março a outubro de 2020. De modo a subsidiar o projeto foram utilizados os seguintes descritores: educação interprofissional, atenção básica, comunidade.

\section{$2^{o}$ Etapa - Construção de ferramentas de educação em saúde a partir da contribuição das práticas colaborativas na Educação Interprofissional do PET-Saúde:}

Visando proporcionar o prosseguimento das atividades do PET-Saúde durante a paralisação de atividades presenciais devido à pandemia pela COVID-19, foram realizadas reuniões via Plataforma Online Google Meet para decidir junto aos coordenadores, tutores, preceptores e alunos os possíveis métodos para a manutenção das produções, visto que é necessária a elaboração de relatórios mensais pela coordenadora geral do projeto, que devem ser encaminhados para o Ministério da Saúde.

Das atividades que foram desenvolvidas, destacam-se temáticas abordadas sobre a COVID-19 e a importância da Atenção Básica como porta de entrada; incentivo à produção de cartilhas, cartazes, folders e outros materiais gráficos sobre a COVID-19, visando os cuidados aos grupos de risco como grávidas, idosos, hipertensos e diabéticos.

Outrossim, eventos on-line também foram desenvolvidos, com destaque ao I Fórum PET-Saúde; I Ciclo de Palestras PET-Saúde; Desigualdade e Racismo Inconstitucional em pauta no SUS; Programa Saúde na Hora: estabelecendo novos horizontes para fazer saúde da família no SUS, dentre outros. 


\section{Resultados e Discussão}

Devido ao contexto pandêmico em que o trabalho foi realizado, o repasse das informações desenvolvidas pelos alunos do Programa PET-Saúde foi feito pelos preceptores e tutores, por meio de cartilhas informativas, nas Unidades Básicas de Saúde em que cada equipe era responsável. Sendo assim, as atividades realizadas foram fundamentais para informar à população a importância de se prevenir, também, contra a contaminação da COVID-19.

A educação em saúde consiste, dentre vários outros aspectos, em aprender sobre doenças, seus efeitos na saúde humana e suas formas de prevenção. O aprendizado, no entanto, também está ligado à promoção da saúde, conforme proposto pela Organização Mundial da Saúde (Santana \& Rossit, 2017).

Convém salientar, ainda, que este trabalho teve dificuldades na devida participação de alguns alunos devido a acessibilidade a internet, pois os encontros eram realizados em plataformas digitais. O que torna-se prepoderante frisar também que as informações desenvolvidas possam chegar em grupos populacionais que se encontram isolados, mas que possuem acesso à internet, e fazer chegar a esta parcela populacional dados e informações cientificamente validadas, confirmando os estudos realizados por Silva, Batistella \& Gomes (2017) e Palácios \& Takenami (2020).

Do mesmo modo, faz-se de suma importância ressaltar o protagonismo da Atenção Básica à Saúde, a qual é a principal responsável pela reformulação e organização do sistema público de saúde e é regulamentada pela Política Nacional de Atenção Básica (PNAB) do Ministério da Saúde (Brasil, 2017). Nesse sentido, esta pesquisa oportunizou reafirmar a importância da Atenção Primária, visto que a divulgação das cartilhas nas Unidades Básicas de Saúde promove maior adesão da comunidade à participação de campanhas e ações sociais, objetivando a prevenção de doenças (Cecílio \& Reis, 2018; Souza, 2017; Rios; Sousa \& Caputo, 2019; Souza, et al., 2017; Vasconcelos, et al., 2017).

Visando o esclarecimento da população acerca da nova doença responsável pela pandemia em curso, a COVID-19, causada pelo SARS-CoV2 (Severe Acute Respiratory Sindrome Coronavirus 2, em tradução livre, "Coronavírus da Síndrome Respiratória Aguda Grave tipo 2), os alunos integrantes do projeto PET-Saúde 2019-2020, sob supervisão de seus preceptores e coordenadores, realizaram pesquisas e trocaram informações acerca da temática, trabalhando estas informações forma interprofissional com a finalidade de fornecer à população geral um apanhado de informações que, ao mesmo tempo completas ao abrangerem as responsabilidades e competências dos cursos dos acadêmicos envolvidos, fossem de fácil acesso e compreensão.

Uma das produções resultantes deste esforço coletivo foi a cartilha intitulada "Lidando com o COVID-19: Dicas para cuidar de si mesmo - e do próximo". A cartilha fornece informações acerca da doença, seu agente etiológico, possíveis sintomas, grupos de risco e ações para redução da propagação. A cartilha incita um aspecto importante: o senso de coletividade e a importância de ações individuais no combate coletivo à pandemia.

É fundamental observar que a linguagem utilizada nas cartilhas é acessível à população, não sendo rebuscada. Além disso, ela destaca dicas para cuidar de si, visto que um dos assuntos mais comentadps na indústria midiática foi sobre a importância da saúde mental em tempos de Pandemia da COVID-19. 
Figuras 1 e 2 - Capa (esquerda) e uma das páginas (direita) da cartilha intitulada "Lidando com o COVID-19: Dicas para cuidar de si mesmo - e do próximo".

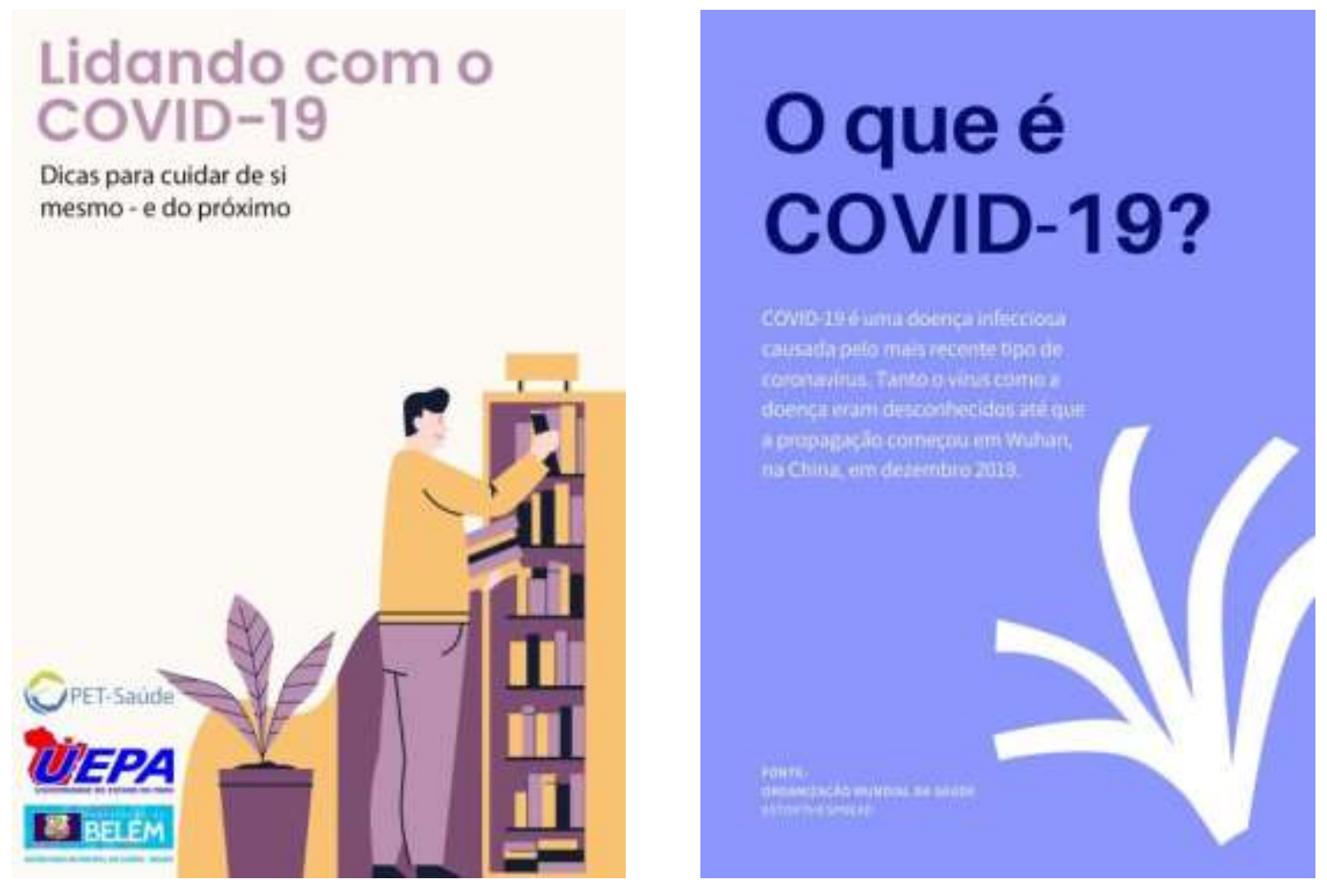

Fonte: Autores.

Tendo em vista o objetivo de manter os grupos de risco, principalmente idosos, em isolamento constante em casa, observou-se também a necessidade de manter os idosos seguros dentro de seus ambientes domésticos. Partindo deste objetivo, realizou-se outra produção coletiva, uma cartilha denominada "Prevenção de quedas: A importância do Planejamento do Ambiente e outras medidas para a manutenção da saúde do idoso em ambiente doméstico", que fornece, de forma acessível, informações sobre comportamentos de risco e formas de prevenção de quedas de idosos em ambientes domésticos.

É oportuno observar que as cartilhas mostram a figura do idoso, chamando a atenção para esse público. 
Figuras 3 e 4 - Capa (esquerda) e uma das páginas (direita) da cartilha intitulada "Prevenção de quedas: A importância do Planejamento do Ambiente e outras medidas para a manutenção da saúde do idoso em ambiente doméstico".
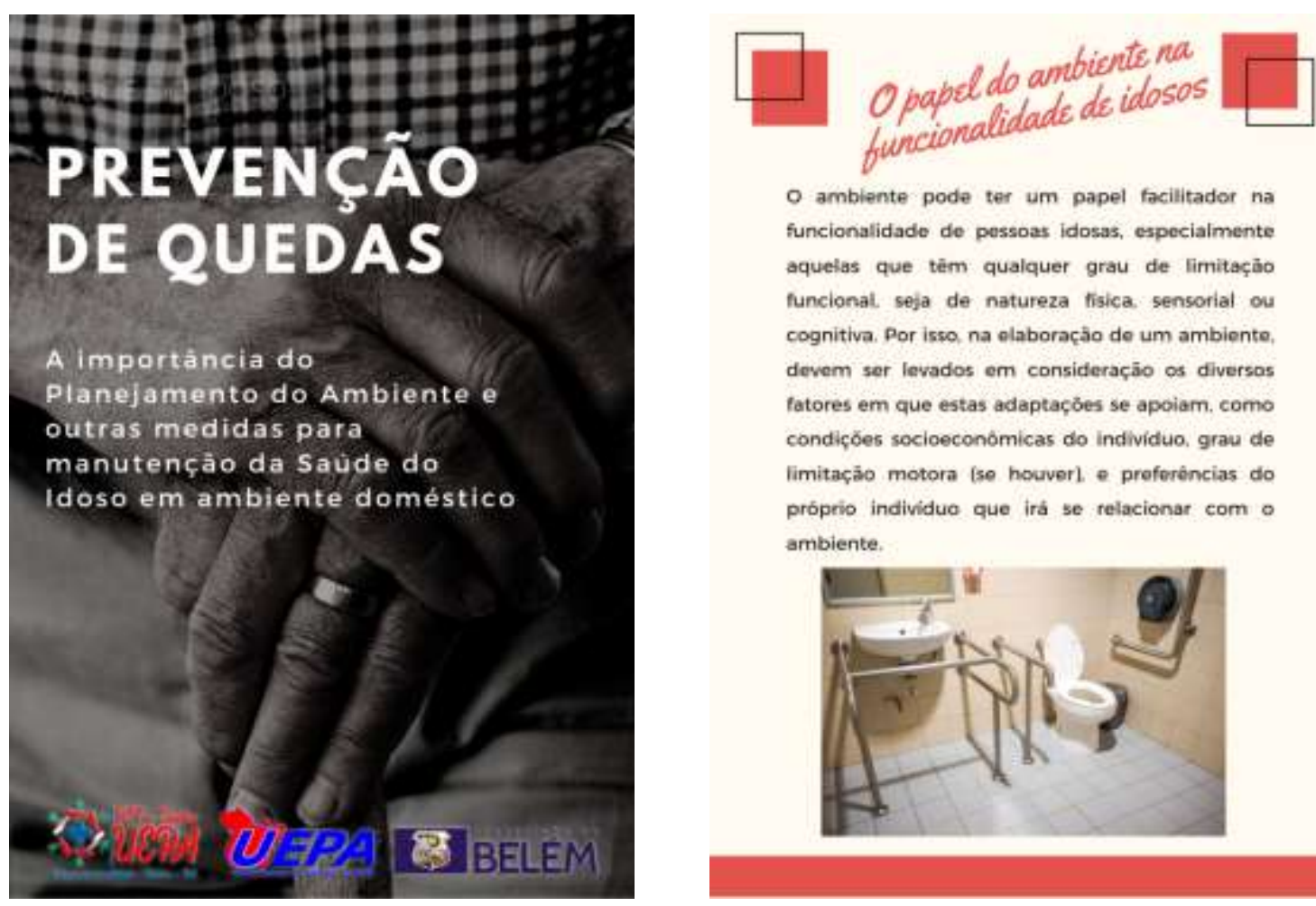

Fonte: Autores.

A construção dessas produções favorece inicialmente uma melhor integração entre docente e seus discentes, na busca de novas ferramentas para a disseminação do conhecimento mesmo em períodos remotos. É importante frisar que todos os envolvidos foram sujeitos ativos na elaboração do material, o que possibilitou aprofundamento e até mesmo incitou outros grupos para a replicação do material.

\section{Conclusão}

A cartilha favoreceu a disseminação de informações seguras, em um período turbulento e permeado de notícias falsas. Assim, buscou-se fontes confiáveis e embasamento científico prioritariamente durante todo processo, além do mais efetivando as práticas colaborativas, como eixo primordial do programa.

As temáticas escolhidas visavam oportunizar a melhoria no acesso as informações que pudessem atingir vários públicos e que também houvesse retorno para a comunidade onde há atuação dos discentes, atingindo um número expressivo do público.

É oportuno mais estudos que possam retratar, também, a opnião da comunidade sobre a importância da divulgação desses materiais a fim de promover a educação em saúde e intregar ainda mais o eixo usuário do SUS e profissionais da Atenção Básica. 


\section{Referências}

Agreli, H. F., Peduzzi, M. \& Silva, M. C. (2016). Patient centred care in interprofessional collaborative practice. Interface, 20 (59), $905-916$.

Araújo, P. C. (2014). Trabalho em equipe multiprofissional na Estratégia Saúde da Família: a interface entre a equipe de Saúde Bucal e a equipe de Saúde da Família. Dissertação [Mestrado Profissional] Fiocruz. Escola Nacional dessaúde Pública Sérgio Arouca.

Batista, C.B. (2013). Movimentos de reorientação da formação em saúde e as iniciativas ministeriais para as universidades. Barbarói, 38, 97-125.

Brasil. Conselho Nacional de Secretários de Saúde. (2011). A Gestão do Trabalho e da Educação na Saúde / Conselho Nacional de Secretários de Saúde. Brasília: CONASS, 120 p.

Brasil. Constituição da República Federativa do Brasil: texto constitucional promulgado em 5 de outubro de 1988, com as alterações determinadas pelas Emendas Constitucionais de Revisão nos 1 a 6/94, pelas Emendas Constitucionais nos 1/92 a 91/2016 e pelo Decreto Legislativo no 186/2008. - Brasília : Senado Federal, Coordenação de Edições Técnicas, 2016. 496 p.

Brasil. Ministério da Saúde. Secretaria de Gestão do Trabalho e da Educação na Saúde. Departamento de Gestão da Educação na Saúde. Política Nacional de Educação Permanente em Saúde: o que se tem produzido para o seu fortalecimento? / Ministério da Saúde, Secretaria de Gestão do Trabalho e da Educação na Saúde, Departamento de Gestão da Educação na Saúde - 1. ed. rev. - Brasília : Ministério da Saúde, 2018. 73 p.

Brasil. Ministério da Saúde. Secretaria de Gestão do Trabalho e da Educação na Saúde. Departamento de Gestão da Educação na Saúde. (2018). Programa de Educação pelo Trabalho para a Saúde: Um panorama da edição PET-Saúde/GraduaSUS / Ministério da Saúde, Secretaria de Gestão do Trabalho e da Educação na Saúde, Departamento de Gestão da Educação na Saúde - Brasília : Ministério da Saúde, 50 p.

Brasil. Ministério da Saúde. (2020). Plano Nacional de Saúde. 1-159.

Cerballos, A. G. C. (2015). Determinação social do processo saúde e doença. In: Cerballos, A. G. C. Modelos conceituais de saúde, determinação social do processo saúde e doença, promoção da saúde. Recife: Grupo saber tecnologias educacionais e sociais UNA-SUS UFPE.

OMS, Organização Mundial de Saúde. Marco para Ação em Educação Interprofissional e Prática Colaborativa. (2010). Gabinete da Rede de Profissões de Saúde - Enfermagem \& Obstetrícia do Departamento. 165 p.

Palácios, M. A. V. \& Takenami, I. (2020). Em tempos de pandemia pela COVID-19: o desafio para a educação em saúde. Vigilância Sanitária em Debate: Sociedade, Ciência \& Tecnologia (Health Surveillance under Debate: Society, Science \& Technology) - Visa Em Debate, 8 (2), 10-15.

Rios, D. R. S., Sousa, D. A. B. \& Caputo, M. C. (2019). Diálogos interprofissionais e interdisciplinares na prática extensionista: o caminho para a inserção do conceito ampliado de saúde na formação acadêmica. Interface (Botucatu), 23.

Farias, D. N., et al. (2018). Interdisciplinaridade e interprofissionalidade na Estratégia Saúde da Família. Trab. Educ. Saúde, 16 (1), 141-162.

Grillo, L. P., Ibarra, L. S. \& Mezadri, T. (2019). Conceito ampliado de saúde na formação dos profissionais de educação física. Saúde e Pesquisa, 12 (2), 265 273.

Lourenço, A. E. P., et al. (2017). Programa de educação pelo trabalho para a saúde (PET-Saúde) e a formação do nutricionista num campus de interiorização. Demetra: alimentação, nutrição \& saúde, 12 (1), 41-58.

Santana, M. C. C. P. \& Rossit, R. A. S. (2017). Interprofissionalismo nas residências multiprofissionais em saúde: análise na região nordeste do Brasil. Journal of management and primary health care, 8 (3), 45-46.

Silva, J. P. V., Batistella, C. \& Gomes, M. L. (2017). Problemas, necessidades e situação de saúde: uma revisão de abordagens para a reflexão e ação da equipe de saúde da família. In: Fonseca, Angélica Ferreira, Corbo, Ana Maria D’Andrea (Org.). O território e o processo saúde-doença. Rio de Janeiro: EPSJV/FIOCRUZ, 2007. (Coleção Educação Profissional e Docência em saúde: a formação e o trabalho do agente comunitário de saúde, p. 159-176.

Souza, C. D. F. (2017). Determinantes sociais de saúde vs determinação social de saúde: uma aproximação conceitual. In: Webcongresso Internacional De Direito Sanitário, I, Anais eletrônicos. Cad. Ibero-Amer. Dir. Sanit., 116-124.

Souza, D. L. B., et al. (2017). Estudos Ecológicos. In: Bedaque, H. P., Bezerra, E. L. M. Descomplicando MBE: uma abordagem prática da medicina baseada em evidências. Caula de Papiro. 29-38.

Vasconcelos, M. I. O., et al. (2017). Educação em saúde na atenção básica: uma análise das ações com hipertensos. Rev. APS, 20 (2), 253-262. 